\title{
Measurements of the director fluctuations in nematics by speckle interference
}

\author{
Marek W. Sierakowski ${ }^{*}$ \\ Faculty of Physics, Warsaw University of Technology, Koszykowa 75, 00-662 Warszawa, Poland
}

Received September 28, 2010; accepted September 30, 2010; published September 30, 2010

\begin{abstract}
Orientational fluctuations in nematics were experimentally investigated by homodyne speckle interference. Thermally excited fluctuations are local stochastic events. Such fluctuations in most cases relax in time aperiodically. In this experiment fluctuations were incidentally observed, and they could oscillate during some life-time before decaying. They were correlated spatially over an area of relatively large size. For convenience, large oscillating fluctuations, correlated in time and space, are called in this report "fluctuons". Usually, the fluctuons observed in the experiment had several oscillating cycles. An average lifetime of single fluctuons measured was a few miliseconds. The fluctuons have thermal origin but their average amplitude and probably also the correlation length could have been enhanced by electric DC, low frequency or optical field (in the linear range), even in the range below the re-orientational threshold intensity.
\end{abstract}

Thermally excited orientational fluctuations in liquid crystals have not been sufficiently explored yet, although they are involved in the majority of optical effects. In most cases, they are a destructive component in LC-based devices, negatively influencing their optical properties and performance [1]. On the contrary, in some applications, like nonlinear light scattering and coherent wave mixing, they play a fundamental role enabling operation. Up to now only the second, advantageous side has been extensively investigated and a number of reports have been published [2]. Few publications report also some attempts to utilize the fluctuations as a tool or an indicator, for instance in surface investigation [3]. However, the basic theory developed for the description of field-induced molecular reorientation in nematics, i.e. the Frank-Oseen equation for static deformation distribution, as well as the Ericksen-Leslie equation for the dynamics of the process, both ignore director fluctuations. This simplification leads to some inconsistency in the theory and discordance with observations in some details. Since fluctuations, in addition, may affect electro-optical characteristics and response times, they cannot be neglected and should be better recognized.

Director fluctuations have been investigated in the nematic cells of simple geometries, both planar (homogeneous) and homeotropic, in order to simplify

*E-mail: sierak@if.pw.edu.pl interpretation of the results. In the reported experiment relative magnitude of fluctuations and their dynamics (time evolution) were measured in thermodynamic equilibrium and also in an electric or optical field. These measurements were carried out in the set-up shown in Fig. 1, by recording a depolarized homodyne speckle interference signal.

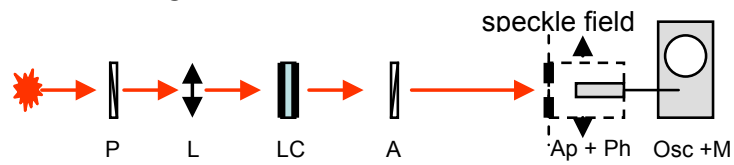

Fig. 1. The experimental lay-out: P, A - polarizers, L - movable lens, LC - liquid crystalline cell, $\mathrm{Ap}+\mathrm{Ph}$ - photomultiplier with diaphragm, Osc $+\mathrm{M}-$ oscilloscope/meter.

A coherent light beam was linearly polarized and then focused on a nematic sample. The beam waist was estimated as low as about $10 \mu \mathrm{m}$. After passing the LCcell placed between crossed polarizers, the beam formed a fluctuating speckle field where a detector was mounted. The samples could rotate over the set-up axis (the "in plane" rotation). The measurements of fluctuations affected by the field were made at a low electric or optic field frequency $(300-1000 \mathrm{~Hz})$.

The speckle image in the far field is produced by the interference of a number of different phasors in the object area illuminated by the laser beam. The signal detected in the interference image depends on both speckle intensity and size (more strictly: the size related to the detector entrance aperture). The size dependence is a result of chaotic phase differences of particular speckles. The smaller are the speckles, the more speckles are gathered by the detector, the better they can compensate mutually, and the smaller is the resulting signal. On the contrary, the signal increases if the speckles in the detector's entrance become correlated in space and time. By similar reasons the speckles intensity, as well as their size, depend, in turn, on the correlation area of the fluctuations (phasors) in relation to the object area. The object area in the experiment was determined by the setup aperture, i.e. by the laser beam spot, which was tunable by the movement of the focusing lens. 
Thermal fluctuations are stochastic events and their magnitude, space distribution and excitation moments chaotically vary in time. As phasors, they produce a dynamic speckle image and cause the detected signal also to vary in time. Any particular fluctuation $\delta$ n of the director in the considered cells can be decomposed into the "in-plane" and the "off-plane" components. The "offplane" component is orthogonal to the cell plane and in terms of the molecular tilt angle $\theta$ can be denoted as $\delta \theta$. In order to check if the variations of the speckleinterference signal really reflect the fluctuation noise, the measuring set-up was examined by several sample orientations. The following simple geometrical analysis shows what is to be expected in particular cases.

Consider a planar nematic layer placed perpendicularly to a light beam between crossed polarizers, like in the experimental set-up. The light is linearly polarized and the optical vector of the incident wave makes the angle $\alpha$ with the average orientation of the fluctuating director in the layer.

At the input, i.e. at the entering plane of the layer, the optical field $\mathrm{E}_{\text {in }}$ is written by standard procedure as a combination of two independently propagating waves, the ordinary $\mathrm{E}_{\mathrm{o}}$ and extraordinary $\mathrm{E}_{\mathrm{e}}$, with:

$E_{e}=\left(E_{\text {in }} \cos \alpha\right) \cos \omega t$, and $E_{o}=\left(E_{\text {in }} \sin \alpha\right) \cos \omega t$.

These components exit the layer with the phase difference

$$
\delta=2 \pi \Delta \mathrm{n}(\mathrm{d} / \lambda)
$$

where $\Delta \mathrm{n}$ is the birefringence of the used LC-material. Therefore at the output the components are:

$\mathrm{E}_{\mathrm{e}}{ }^{\prime}=\left(\mathrm{E}_{\mathrm{e}}^{\mathrm{m}}\right) \cos \omega \mathrm{t}^{\prime}$, and $\mathrm{E}_{\mathrm{o}}{ }^{\prime}=\left(\mathrm{E}_{\mathrm{o}}{ }^{\mathrm{m}}\right) \cos \left(\omega \mathrm{t}^{\prime}+\delta\right)$.

From the above one can readilly find the resulting field intensity in the orthogonal polarization ${ }^{+} \mathrm{E}_{\text {out }}$ at the output plane as:

$$
\left|{ }^{+} E_{\text {out }}\right|=\left(E_{\text {in }} \sin 2 \alpha \sin 1 / 2 \delta\right) \sin \omega \mathrm{t}^{\prime},
$$

where $t^{\prime} \prime=\left[t^{\prime}-(1 / 2 \delta / \omega)\right]$.

The amplitude term of $\mid{ }^{+} \mathrm{E}_{\text {out }}$ (in brackets) depends both on the cell orientation $\alpha$ and on the phase delay $\delta$. The sensivity of the $\left|{ }^{+} E_{\text {out }}\right|$ on the "in plane" fluctuation $d \alpha$ is then:

$$
\sigma_{\alpha}=\left|\frac{\partial^{+} E_{\text {out }}}{\partial \alpha}\right|=2 E_{\text {in }} \cos 2 \alpha \sin \frac{\delta}{2}
$$

and becomes:

$\sigma_{\alpha}=0$ for $\alpha=\pi / 4$ and any $\delta$,

$\sigma_{\alpha}=$ maximum for $\alpha=0, \pi / 2$,

varying with $\delta$ like $\sin (\delta / 2)$ to wit:

$\sigma_{\alpha}=0 \quad$ for $\delta=2 \pi \mathrm{n} \quad(\lambda$-plate $)$,

$\sigma_{\alpha}=$ maximum for $\delta=(2 n+1) \pi(\lambda / 2-$ plate $)$.

So, the "in plane" fluctuations should yield no signal (or, in real conditions small) for the cell orientation $\alpha=\pi / 4$, and oscillating one for $\alpha=0, \pi / 2$, with minima and maxima, when $\delta$ varies.

In turn, the sensitivity of the output intensity on the "off plane" fluctuations $\mathrm{d} \theta$ is:

$$
\sigma_{\theta}=\left|\frac{\partial^{+} E_{\text {out }}}{\partial \theta}\right|=\left|\frac{\partial^{+} E_{\text {out }}}{\partial \delta} \| \frac{\partial \delta}{\partial \theta}\right|
$$

The function $\delta(\theta)$ is given by Eq.(1) with $\Delta \mathrm{n}(\theta)$ as in [4]:

$$
\Delta n(\theta)=n_{e f}(\theta)-n_{o}=\frac{n_{o} n_{e}}{\sqrt{n_{e}{ }^{2} \sin ^{2} \theta+n_{o}{ }^{2} \cos ^{2} \theta}}-n_{o}
$$

From the above one can obtain:

$$
\begin{aligned}
& \left|\frac{\partial^{+} E_{\text {out }}}{\partial \delta}\right|=E_{\text {in }} \sin 2 \alpha \cos \frac{\delta}{2}, \\
& \left|\frac{\partial \delta}{\partial \theta}\right|=\frac{2 \pi d}{\lambda} \frac{\Delta \varepsilon}{n_{e} n_{o}} n_{e f}{ }^{3} \sin 2 \theta
\end{aligned}
$$

Again, the "off plane" sensitivity depends also on $\alpha$ and $\delta$ value. The $\alpha$-dependence in the first expression of (3) shows:

$\sigma_{\theta}=0$ for $\alpha=0, \pi / 2$,

$\sigma_{\theta}=$ maximum for $\alpha=\pi / 4$.

Whereas the $\delta$-dependence gives oscillations with:

$\sigma_{\theta}=$ maximum for $\delta=2 \mathrm{n} \pi \quad(\lambda$-plate $)$,

$\sigma_{\theta}=0 \quad$ for $\delta=(2 n+1) \pi(\lambda / 2-$ plate $)$,

which follow the $\delta$-increase.

By comparing Eqs. (3) with the $\delta$-dependence of the electro-optical transmission (Eq. (2)) in the considered arrangement one can notice that minima and maxima of these two curves should be shifted by $\Delta \delta=\pi / 2$.

The second term of $\sigma_{\theta}$ (in Eq. (3)) is a function of the molecular re-orientation angle $\theta$ via $\mathrm{n}_{\mathrm{ef}}$ and $\sin 2 \theta$. The deformation $\theta$ for a planar sample obeys $0<\theta<\pi / 2$. In this range, for a positive-birefringence material, the effective index $\mathrm{n}_{\mathrm{ef}}$ decreases monotonically from $\mathrm{n}_{\mathrm{e}}$ to $\mathrm{n}_{\mathrm{o}}$ while $\sin 2 \theta$ imposes on it two zeroing points for $\theta=0, \pi / 2$.

To summarize, the "off plane" sensitivity $\sigma_{\theta}$, in contrast to the "in plane" sensitivity $\sigma_{\alpha}$, should follow the electro-optical dependence with some shift at $\alpha=\pi / 4$ and should vanish at $\alpha=0, \pi / 2$, and $\theta=0, \pi / 2$.

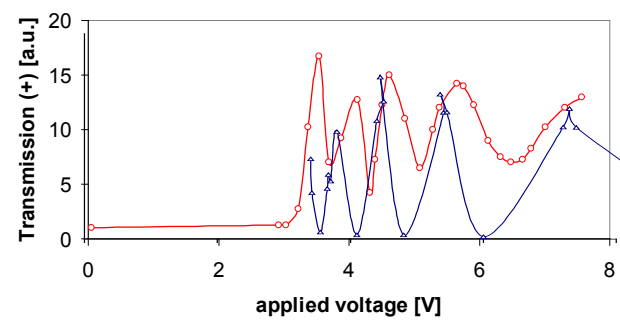

Fig. 2. Comparison of voltage dependence of electro-optical transmission ( $r e d$ ) with detected signal variations in the speckle field (blue curve).

The measurements carried out for a planar cell showed a negligibly small signal just for $\alpha=0, \pi / 2$, and a considerably large one for $\alpha=\pi / 4$. After the voltage was applied to the cell and the re-orientation was progressed, the signal revealed minima and maxima. As it can be seen 
in Fig. 2, the signal is shifted with respect to electrooptical transmission.

The signal faded for a large deformation and fully disappeared for homeotropic orientation $(\theta=\pi / 2)$. The small initial tilt angle and imperfections of the cell alignment might be responsible for the non-vanishing signal at $\theta=0$ (i.e. with no field applied).

In view of the preceding discussion, the above results support the conclusion that the observed signal is generated prevailingly by $\theta$-fluctuations so that the "in plane" - d $\alpha$ - is not seen here. This seems likely because the distortions $d \alpha / d z$ (i.e. fluctuations having a wave vector along the light propagation) are related to the twist deformation of the structure. These distortions, by fixed molecular orientation at both boundaries, do not change the polarization plane of entering light, provided that the wave vector $\mathrm{q}$ is still in the Maguin limit $(1 / \mathrm{q} \gg \lambda)$. The light is then shut off by the analizer in the set-up. It should be noted that mainly the measured fluctuations, $\mathrm{d} \theta / \mathrm{dz}$, may affect the field-induced re-orientation process $\theta(z)$.

Thermally excited fluctuations mostly relax exponentially with the decay time $\tau_{d}$ which was observed here to be of the order of miliseconds. It corresponds to a light scattering frequency of several hundreds $\mathrm{Hz}$, in agreement with literature data $[5,9]$. However, in this experiment, a sudden increase of the recorded signal was incidentally observed, followed by several cycles of oscillations before it exponentially decayed. Figure 3 shows the record of such an event, indicating the possibility of an incidental, significant increase of the correlation area of a fluctuation over some average size.

Such a large, oscillating fluctuation forms a local temporal distortion which can be considered as a separate stochastic event; for convenience let us call it "the fluctuon". If the fluctuon is sufficiently large, it may be expected to react to an applied field.

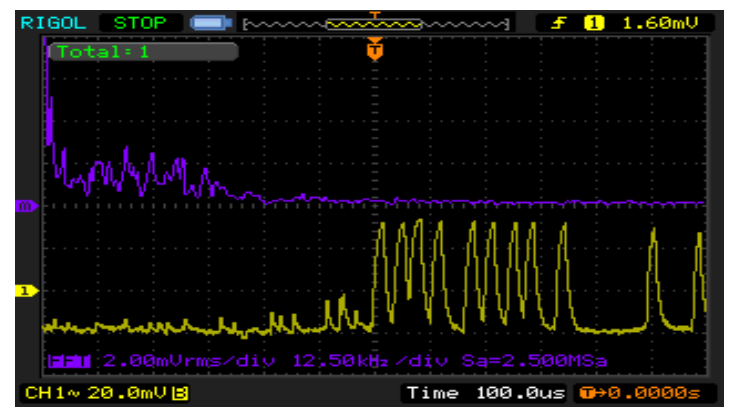

Fig. 3. The oscilloscope record of the speckle intensity showing an incidental increase of fluctuation noise. Temporarily oscillating signal peaks are also seen. No field was applied.

The effects of quenching (freezing) $[6,7]$ or stimulating $[2,8]$ of orientational fluctuations in an intense field are known. Here, field enhancement of fluctuations was observed by a relatively low field in the sub-threshold range before molecular re-orientation could start. Figure 4 presents the measurements of the average amplitude of the detected signal in the LF-electric field below the threshold intensity. The fluctuations increase with the field rise before re-orientation begins. Similar behavior was observed here in the optic field as well. This effect can also be noticed in measurements made by a different technique in other reports [9].

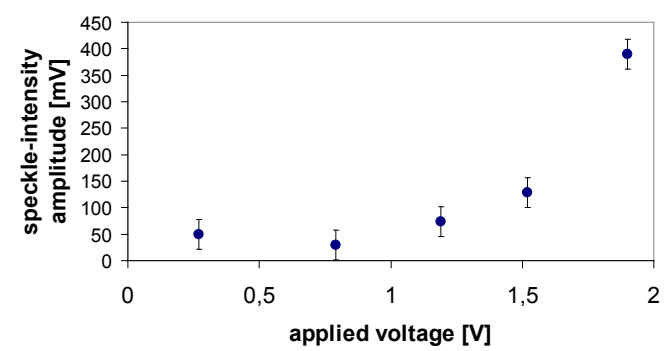

Fig. 4. Average amplitude of speckle intensity versus applied field below the re-orientation threshold which was $1.8 \mathrm{~V}$ in this case.

To conclude, the obtained results point out the presence of oscillating thermal fluctuations of the nematic director. Since no oscillatory movement of orientational fluctuations was detected earlier by light scattering measurements, its contribution to fluctuation statistics is probably not large enough to be visible there. Also, an electric field enhancement of fluctuation magnitude was observed for fields below the reorientational threshold intensity. The used measurement technique does not allow for determining if the field increases only fluctuations amplitude or their correlation length too. Thermodynamic considerations, however, show that an enlargement of the correlation area is feasible. The impact of the observed effects on the field-induced molecular reorientation process is highly predictable.

\section{References}

[1] see for example M. Yoneya, Y. Utsumi, Y. Umeda, J. Appl. Phys. 98, 16160 (2005).

[2] I.C. Khoo, Y. Liang, Phys. Rev. E 65, 5 (2000) and references therein.

[3] J. Papanek, Mol. Cryst. Liq. Cryst. 179, 139 (1990).

[4] P. Oswald, P. Pieranski, Nematic and Cholesteric Liquid Crystals (Taylor and Francis, London, 2005).

[5] I.C. Khoo, S.T. Woo, Optics and nonlinear optics of liquid crystals, (World Scientific Publishing, 1993).

[6] J.C. Filipini, Phys. Rev. Lett. 39, 150 (1977).

[7] P.G. de Gennes, The physics of Liquid Crystals, (Clarendon, Oxford 1973).

[8] N.V. Tabiryan, A.V. Sukhov, B.Ya. Zeldovich, J. Opt. Soc. Am B 18, 8 (2001).

[9] P. Galatola, J.Phys.II France, 21509 (1992). 